\title{
EXTENDED EXTINCTION PROFILE FOR THE CLASSIFICATION OF HYPERSPECTRAL IMAGES
}

\author{
Pedram Ghamisi ${ }^{1,2}$, Roberto Souza ${ }^{3}$, Jon A. Benediktsson ${ }^{4}$, Xiao Xiang Zhu ${ }^{1,2}$, \\ Letícia Rittner ${ }^{3}$ and Roberto Lotufo ${ }^{3}$ \\ ${ }^{1}$ German Aerospace Center (DLR), Remote Sensing Technology Institute (IMF) \\ ${ }^{2}$ Technische Universität München (TUM), Signal Processing in Earth Observation \\ ${ }^{3}$ School of Electrical and Computer Engineering - UNICAMP \\ ${ }^{4}$ Faculty of Electrical and Computer Engineering, University of Iceland
}

\begin{abstract}
In this paper, a novel approach is proposed for the spectralspatial classification of hyperspectral images. The proposed classification approach is based on a novel filtering technique, here entitled as extended extinction profile (EEP). The proposed classification approach is applied on two well-known data sets: Pavia University and Indian Pines; and the obtained results have been compared with one of the strongest filtering approaches in the literature named extended attribute profile (EAP). Results confirm that the proposed approach is able to efficiently extract spatial information for the classification of hyperspectral images.
\end{abstract}

Index Terms - Extinction Profile, hyperspectral Image Classification, Support Vector Machine.

\section{INTRODUCTION}

The classification of hyperspectral images by considering both spectral and spatial information has become a vibrant topic of research recently. The inclusion of spatial information into a classification framework can significantly improve the quality of the final classification map in particular for very high resolution hyperspectral data.

In order to efficiently exploit spatial information, a wide variety of techniques based on crisp and adaptive neighborhood systems have been proposed in the literature. A comprehensive information regarding different techniques for the spectral-spatial classification of hyperspectral data can be found in [1].

In 2001 [2], the concept of morphological transformations was taken into account to build the so-called morphological profile (MP). Then, in [3], the concept of MPs was successfully applied on hyperspectral data leading to the so-called

This research has been partly supported by Alexander von Humboldt Fellowship for postdoctoral researchers, Helmholtz Young Investigators Group "SiPEO" (VH-NG-1018, www.sipeo.bgu.tum.de) and FAPESP grants 2013/23514-0, 2015/12127-0. extended morphological profile (EMP). Since then, the EMP and its modifications have been enormously used as a tool for further improvement of classification accuracies for hyperspectral data. However, the concept of an MP has a few shortcomings such as (i) the shape of SEs is fixed and (ii) SEs are not able to characterize information related to the graylevel characteristics of the regions. In 2010, the concept of attribute profile (AP) was introduced in order to address such shortcomings [4]. A comprehensive survey on APs and its capabilities for the classification of remote sensing data can be found in $[5,1]$.

To further improve the classification accuracies of EAP, in this paper, a novel filtering profile is proposed for the classification of hyperspectral data. The approach is based on the concept of extinction values [6] here known as extended extinction profile (EEP). EEP simultaneously simplify the input image by discarding unimportant spatial details and preserves the geometrical characteristics of the other regions from the first informative features extracted by an Independent Component Analysis (ICA) on a hyperspectral data set. In addition, the proposed approach is fully automatic and it can adjust the filtering parameters based on the number of extrema. The output of this step provides a few informative features, which can be fed to a support vector machine (SVM) classifier. In this paper, SVM is considered for classification since it can handle high dimensional data with even limited number of training samples and its capability has been already proven for the classification of MPs and APs [5]. Finally, the obtained results are compared with extended AP (EAP) in terms of classification accuracies on two hyperspectral data sets; Indian Pines and Pavia University. Results confirm the capability of EEPs for the classification of hyperspectral data.

The rest of the paper is organized as follows: Section 2 is devoted to the EEP methodology. Section 3 presents experimental results on two well-known data sets. Section 4 wraps up the paper by providing the main concluding remarks. 


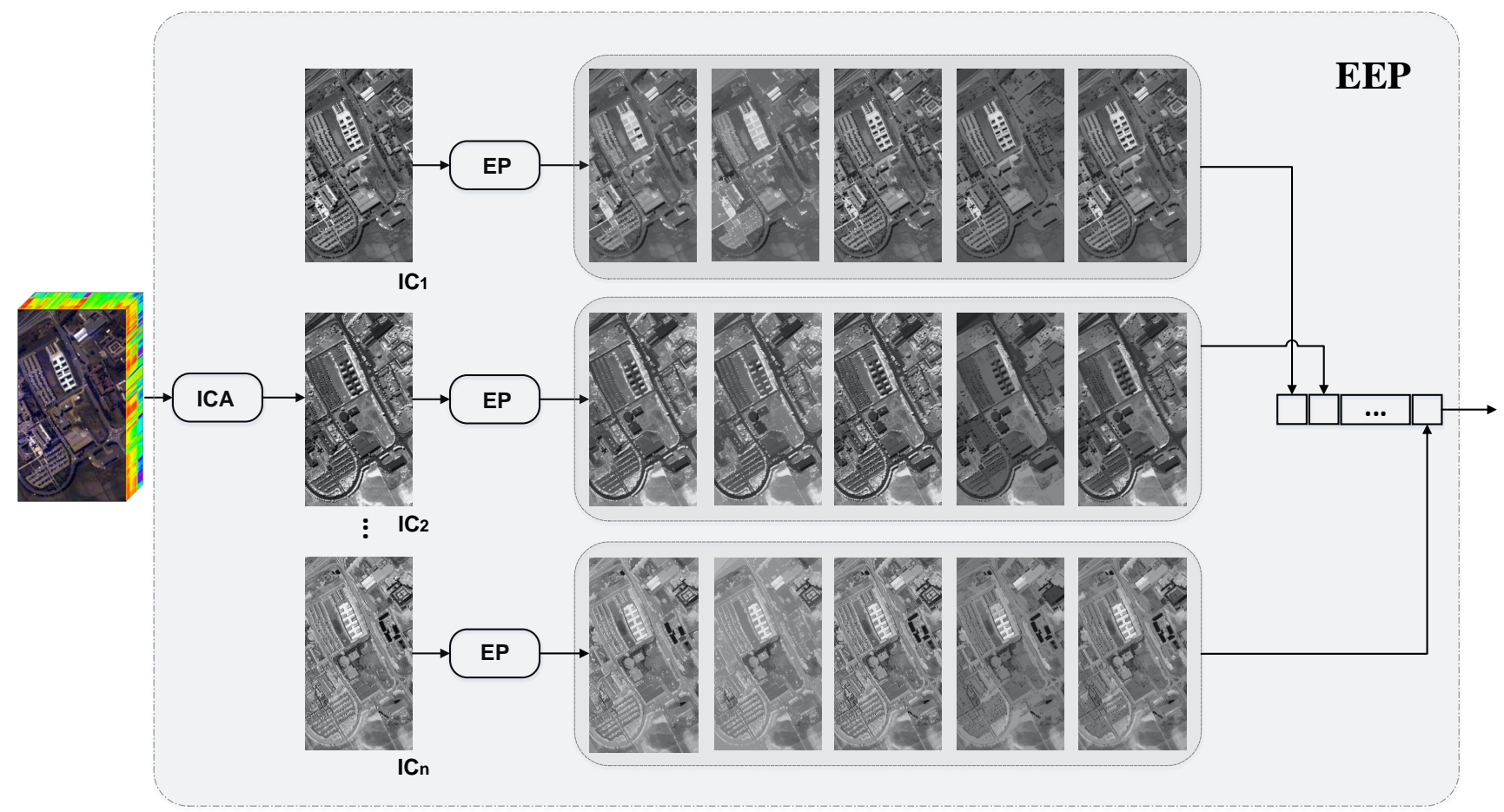

Fig. 1: EEP general architecture. ICA is applied to the hyperspectral data. Then, the the EP is computed for the most informative principal components. Finally, EPs are concatenated to generate the EEP.

\section{METHODOLOGY}

Fig. 1 illustrates the general architecture of the proposed approach (EEP). As can be seen, first, ICA is applied on the input data and the first informative ICs (here, we retained the first three components for both data sets). Then, EP is performed on the most informative ICs. The produced features are concatenated into a stacked vector and classified by an SVM. More details are given in the following subsections.

\section{EXTICTION FILTER}

Extinction filter is a connected filter that preserves the relevant extrema of the image. It can be defined as follows: consider that $\operatorname{Max}(f)=\left\{M_{1}, M_{2}, \ldots, M_{N}\right\}$ is the set of regional maxima of the image $f$. Each regional maxima $M_{i}$ has an extinction value $\epsilon_{i}$, conformed defined by Vachier [6]. The extinction filter $(\mathrm{EF})$ of $f$ set to preserved the $n$ maxima with highest extinction values is given by:

$$
E F^{n}(f)=R_{g}^{\delta}(f),
$$

where $R_{g}^{\delta}(f)$ is the reconstruction by dilation [7] of the mask image $g$ from marker image $f$. The mask imae $g$ is given by:

$$
g=\max _{i=1}^{n}\left\{M_{i}^{\prime}\right\},
$$

where max is the pixel-wise maximum operation. $M_{1}^{\prime}$ is the maximum with highest extinction value, $M_{2}^{\prime}$ has the second highest extinction value, and so on.

Extinction filters can be efficiently implemented in the max-tree structure [8]. The $n$ maxima (max-tree leaves) with highest extinction values concerning the attribute being analyzed are chosen. The nodes in the paths from these leaves to the the root are marked as to be kept. All other nodes are pruned from the max-tree.

Extinction filters are closely related to dynamic openings [9], and unlike the usual contrast and size filters the heights of the remaining extrema in the image are completely preserved. They are useful to discern relevant from irrelevant extrema in the image. Souza et al. showed that extinction filters are a more efficient alternative than attribute filters with respect to simplification for recognition [8], since it is able to preserve more regions and correspondences found by affine region detectors. Another advantage of extinction filters over attribute filters is that extinction filters parameters are easier to be set, independently of the kind of attribute being used (e.g. area, volume,...), since they are based on the number of extrema. The thresholds used by attribute filters vary greatly according to the attribute being used as well as the data set being analyzed, therefore are more difficult to be set. In other words, the main shortcoming of conventional APs which are related to the initialization of the threshold values is being addressed in EPs. 


\section{EXTINCTION PROFILE AND ITS EXTENSION TO HYPERSPECTRAL DATA}

In order to extract more detailed information from input images, instead of applying one filtering step, a sequence of filtering steps with progressively higher threshold values can be taken into account. In this context, EPs can be achieved. An EP is composed of a sequence of opening and closing transformations defined with a sequence of progressively stricter criteria. An EP for the input gray scale image, $f$, can be defined as in Eq. (3): with $P_{\lambda}:\left\{P_{\lambda_{i}}\right\}(i=1, \ldots, L)$ a set of $L$ ordered predicates (i.e., $P_{\lambda_{i}} \subseteq P_{\lambda_{k}}, i \leq k$ ). For EPs, the number of extrema can be considered as the predicates.

In order to generalize the concept of EP (which is originally developed for gray scale images) to hyperspectral data, one possible way is based on performing a feature reduction approach like PCA or ICA and applying EPs on the most informative features [1]. In more detail, this approach is based on the reduction of the dimensionality of the image from $E \subseteq$ $\mathbf{Z}^{n}$ to $E^{\prime} \subseteq \mathbf{Z}^{m}(m \leq n)$ with a generic transformation $\Psi: E \rightarrow E^{\prime}$ applied to an input image $\mathbf{f}$ (i.e., $\mathbf{q}=\Psi(\mathbf{f})$ ). Then, EP can be applied on the most informative features $g_{i}$ $(i=1, \ldots, m)$ of the transformed image. This can be mathematically described as:

$$
E E P(\mathbf{q})=\left\{E P\left(q_{1}\right), E P\left(q_{2}\right), \ldots, E P\left(q_{m}\right)\right\} .
$$

\section{EXPERIMENTAL RESULTS}

In this paper, two well-known hyperspectral data sets; Indian Pines and Pavia University have been taken into account. In order to make the proposed approach fully comparable with the state-of-the-arts, we have used exactly the same number of test and training samples as reported in [10].

ICA is initially applied on the above-mentioned data sets and the first three ICs are kept. More ICs can be also considered. Here, SVM was considered for the classification step as it can handle highly redundant features produced by either EAP or EEP in a proper way [1]. For the SVM, a Gaussian kernel is taken into account and the hyper-tuning parameters are selected using five fold cross-validation. Here, the areaopen filter [11] has been taken into account to generate the APs using the thresholds $\{25,100,500,1000,5000,10000$, $20000,50000,100000,150000\}$. In order to generate the EP, the area extinction filter was considered. The values of $n$ used to generate the profile are automatically given by the following equation:

$$
\left\lfloor 2^{j}\right\rfloor j=0,1, \ldots, s-1 .
$$

The total EP size is $2 s+1$, since the original image is also included in the profile. We set $s=10$ for having the same number of features for both $\mathrm{AP}$ and $\mathrm{EP}$ in order to have a fair comparison. The profiles were computed considering the 4-connected connectivity rule.
As can be seen in Table 1, for both data sets, both AP and EP significantly outperformed the SVM, which confirms the capability of using AP and EP for the classification of hyperspectral data. In general, the EP has improved the AP in terms of classification accuracies for both data sets due to its capability to preserve more relevant regions suitable for classification. The second reason is that extinction filters preserve the height of the extrema which is useful for discriminating different classes of interest. Figures 2 and 3 illustrate the outputs of classification approaches by considering (a) an SVM on the input data, (b) EAP and (c) EEP for Pavia University and Indian Pines, respectively. As can be seen, for both data sets, the consideration of spatial information can highly improve the quality of the classification map by reducing the salt and pepper appearance of labeled pixels and the uncertainty of labels when there is no spatial information injected to the classifier.

\section{CONCLUSION}

In this paper, a novel approach has been proposed for the classification of hyperspectral data, which is based on extinction filters here entitled as extended extinction profile. The new approach has been applied on two well-known hyperspectral data sets and the obtained results have been compared with EAP and SVM. With respect to the experiments, two main conclusions can be obtained: (1) extinction profiles can outperform attribute profiles in terms of classification accuracies due to its capability to preserve more relevant regions as well as preserving the height of the extrema, and (2) it works naturally with the number of extrema, which seems to provide better results in terms of classification accuracies and decreases the burden of setting threshold values. It should be noted that the proposed approach can be considered as a template and different parts of that such as ICA and SVM can be replaced by more advanced approaches.

\section{REFERENCES}

[1] J. A. Benediktsson and P. Ghamisi, Spectral-Spatial Classification of Hyperspectral Remote Sensing Images, Artech House Publishers, INC, Boston, USA, 2015.

[2] M. Pesaresi and J. A. Benediktsson, "A new approach for the morphological segmentation of high-resolution satellite imagery," IEEE Trans. Geos. Remote Sens., vol. 39, no. 2, pp. 309-320, 2001.

[3] J. A. Benediktsson, J. A. Palmason, and J. R. Sveinsson, "Classification of hyperspectral data from urban areas based on extended morphological profiles," IEEE Trans. Geos. Remote Sens., vol. 43, no. 3, pp. 480-491, 2005.

[4] M. Dalla Mura, J. A. Benediktsson, B. Waske, and L. Bruzzone, "Morphological attribute profiles for the 


$$
E P(f)=\{\underbrace{\phi^{P_{\lambda_{L}}}(f), \phi^{P_{\lambda_{L-1}}}(f), \ldots, \phi^{P_{\lambda_{1}}}(f)}_{\text {thickening profile }}, f, \underbrace{\gamma^{P_{\lambda_{1}}}(f), \ldots, \gamma^{P_{\lambda_{L-1}}}(f), \gamma^{P_{\lambda_{L}}}(f)}_{\text {thinning profile }}\},
$$

Table 1: SVM: Classification results of Indian Pines and Pavia University data sets by considering AP and EP with parameters defined in Section 3.1. Metrics AP and EP are reported in percentage. Kappa is a coefficient with changes in the range of 0 and 1. Best results for each data set is bold. SVM refers to a situation where the original data have been classified by an SVM classifier.

\begin{tabular}{|c|c|c|c|c|c|c|}
\hline & \multicolumn{3}{|c|}{ Indian Pines } & \multicolumn{3}{c|}{ Pavia University } \\
\hline Metric & SVM & AP & EP & SVM & AP & EP \\
\hline OA(\%) & 73.39 & 89.29 & $\mathbf{9 1 . 2 2}$ & 78.21 & $\mathbf{9 7 . 0 5}$ & 96.16 \\
\hline AA(\%) & 82.76 & 92.75 & $\mathbf{9 3 . 9 3}$ & 87.14 & 95.70 & $\mathbf{9 6 . 1 9}$ \\
\hline Kappa & 0.7002 & 0.8777 & $\mathbf{0 . 8 9 9 5}$ & 0.7333 & 0.9436 & $\mathbf{0 . 9 4 9 4}$ \\
\hline
\end{tabular}

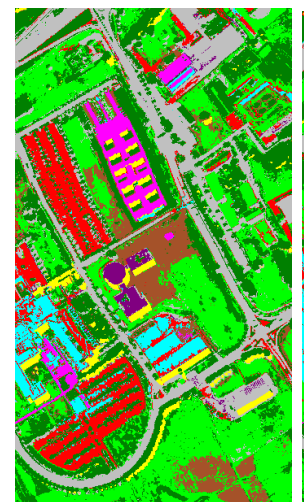

(a)

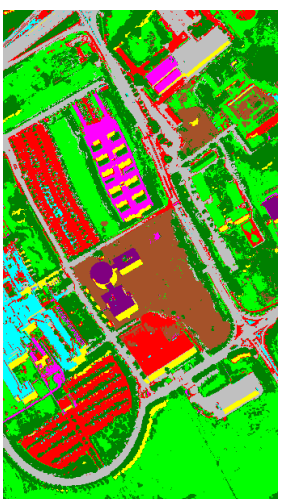

(b)

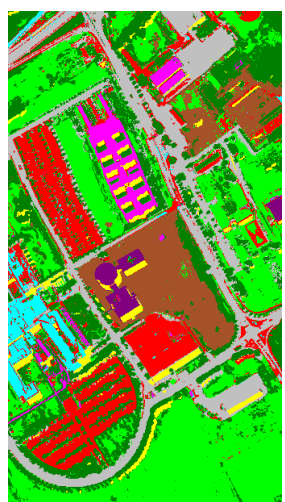

(c)
Fig. 2: The classification maps for Pavia University by considering (a) SVM on the input data (b) EAP (c) EEP.

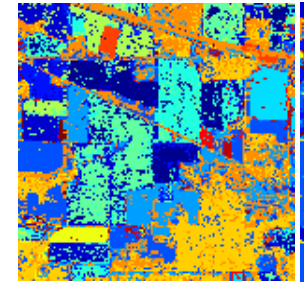

(a)

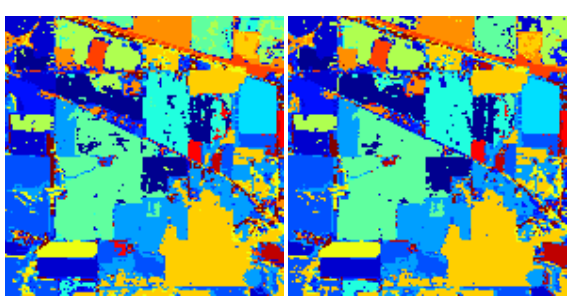

(b) (c)
Fig. 3: The classification maps for Indian Pines by considering (a) SVM on the input data (b) EAP (c) EEP. analysis of very high resolution images," IEEE Trans. Geos. Remote Sens., vol. 48, no. 10, pp. 3747 - 3762, 2010.

[5] P. Ghamisi, M. Dalla Mura, and J. A. Benediktsson, "A survey on spectral-spatial classification techniques based on attribute profiles," IEEE Trans. Geos. Remote Sens., vol. 53, no. 5, pp. 2335-2353, 2015.

[6] C. Vachier, "Extinction value: a new measurement of persistence," in IEEE Workshop on Nonlinear Signal and Image Processing, 1995, vol. I, pp. 254-257.

[7] Pierre Soille, Morphological Image Analysis: Principles and Applications, Springer-Verlag New York, Inc., Secaucus, NJ, USA, 2 edition, 2003.

[8] R. Souza, L. Rittner, R. Machado, and R. Lotufo, "A comparison between extinction filters and attribute filters," in ISMM'15, Reykjavik, Iceland, May 27-29, 2015. Proceedings, 2015, pp. 63-74.

[9] P. Salembier and M. Wilkinson, "Connected operators," IEEE Signal Processing Magazine, vol. 26, no. 6, pp. 136-157, 2009.

[10] P. Ghamisi, J. A. Benediktsson, G. Cavallaro, and A. Plaza, "Automatic framework for spectral-spatial classification based on supervised feature extraction and morphological attribute profiles," IEEE Journal of Selected Topics in Applied Earth Observations and Remote Sensing, vol. 7, no. 6, pp. 2147-2160, 2014.

[11] L. Vincent, "Morphological area openings and closings for grey-scale images," in Shape in Picture, Ying-Lie O, Alexander Toet, David Foster, HenkJ.A.M. Heijmans, and Peter Meer, Eds., vol. 126, pp. 197-208. Springer Berlin Heidelberg, 1994. 